Directeur de LA publication / Publication diRECTOR: Bruno David

Président du Muséum national d'Histoire naturelle

RÉDACTRICE EN CHEF / EDITOR-IN-CHIEF: Laure Desutter-Grandcolas

ASSISTANTE DE RÉDACTION / AsSISTANT EDITOR: Anne Mabille (zoosyst@mnhn.fr)

Mise en PAge / PAge LAYOUt: Anne Mabille

COMITÉ SCIENTIFIQUE / SCIENTIFIC BOARD:

James Carpenter (AMNH, New York, États-Unis)

Maria Marta Cigliano (Museo de La Plata, La Plata, Argentine)

Henrik Enghoff (NHMD, Copenhague, Danemark)

Rafael Márquez (CSIC, Madrid, Espagne)

Peter $\mathrm{Ng}$ (University of Singapore)

Jean-Yves Rasplus (INRA, Montferrier-sur-Lez, France)

Jean-François Silvain (IRD, Gif-sur-Yvette, France)

Wanda M. Weiner (Polish Academy of Sciences, Cracovie, Pologne)

John Wenzel (The Ohio State University, Columbus, États-Unis)

COUVERTURE / COVER:

Spécimen de Clarotes laticeps (Rüppell, 1829) photographié pendant l'échantillonage (photographie : Amani Reine Élisabeth Kouadio).

Zoosystema est indexé dans / Zoosystema is indexed in:

- Science Citation Index Expanded (SciSearch $\left.{ }^{\circledR}\right)$

- ISI Alerting Services ${ }^{\circledR}$

- Current Contents ${ }^{\circledR} /$ Agriculture, Biology, and Environmental Sciences ${ }^{\circledR}$

- Scopus ${ }^{\circledR}$

Zoosystema est distribué en version électronique par / Zoosystema is distributed electronically by:

- BioOne ${ }^{\circledR}$ (http://www.bioone.org)

Les articles ainsi que les nouveautés nomenclaturales publiés dans Zoosystema sont référencés par / Articles and nomenclatural novelties published in Zoosystema are referenced by:

- ZooBank ${ }^{\circledR}$ (http://zoobank.org)

Zoosystema est une revue en flux continu publiée par les Publications scientifiques du Muséum, Paris / Zoosystema is a fast track journal published by the Museum Science Press, Paris

Les Publications scientifiques du Muséum publient aussi / The Museum Science Press also publish:

Adansonia, Geodiversitas, Anthropozoologica, European Journal of Taxonomy, Naturae, Cryptogamie sous-sections Algologie, Bryologie, Mycologie.

Diffusion - Publications scientifiques Muséum national d'Histoire naturelle

CP $41-57$ rue Cuvier F-75231 Paris cedex 05 (France)

Tél.: 33 (0)1 40794805 / Fax: 33 (0)1 40793840

diff.pub@mnhn.fr / https://sciencepress.mnhn.fr

(C) Publications scientifiques du Muséum national d'Histoire naturelle, Paris, 2021

ISSN (imprimé / print): 1280-9551/ ISSN (électronique / electronic): 1638-9387 


\section{Deux nouveaux Protoancylodiscoides Paperna, 1969 (Monogenea, Ancyrocephalidae) parasites branchiaux de Clarotes laticeps (Rüppell, 1829) (Siluriformes, Claroteidae) en Côte d'Ivoire}

Enoutchy Fabrice BOUAH Valentin N'DOUBA

Laboratoire des Milieux Naturels et Conservation de la Biodiversité, UFR Biosciences, Université Felix Houphouët Boigny, 22 boîte postale 582, Abidjan 22 (Côte d'Ivoire)

fabricebouah@gmail.com ndouval@hotmail.com

Antoine PARISELLE

ISEM, CNRS, Université de Montpellier, IRD, Montpellier (France) et Laboratory "Biodiversity, Ecology and Genome", Mohammed V University in Rabat, Faculty of Sciences, 4 avenue Ibn Batouta, boîte postale 1014, Rabat (Maroc) antoine.pariselle@ird.fr

Soumis le 23 septembre 2020 | Accepté le 3 décembre 2020 | Publié le 30 mars 2021

Bouah E. F., N'Douba V. \& Pariselle A. 2021. - Deux nouveaux Protoancylodiscoides Paperna, 1969 (Monogenea, Ancyrocephalidae) parasites branchiaux de Clarotes laticeps (Rüppell, 1829) (Siluriformes, Claroteidae) en Côte d'Ivoire. Zoosystema 43 (9): 155-162. https://doi.org/10.5252/zoosystema2021v43a9. http://zoosystema.com/43/9

RÉSUMÉ

L'étude des monogènes parasites branchiaux de 20 spécimens de Clarotes laticeps (Rüppell, 1829) (Siluriformes, Claroteidae) capturés avec des filets maillants dans la rivière Bagoué (Côte d'Ivoire) a révélé la présence de deux nouvelles espèces du genre Protoancylodiscoides Paperna, 1969 : Protoancylodiscoides essetchii n. sp. et Protoancylodiscoides ivoiriensis n. sp. Ces nouvelles espèces diffèrent des autres espèces de Protoancylodiscoides déjà décrites sur des hôtes de la famille des Claroteidae Risch, 1986 par la morphologie de l'organe copulateur pour P. essetchii n. sp. et dans la morphologie des crochets dorsaux et ventraux pour $P$. ivoiriensis n. sp. Protoancylodiscoides essetchii n. sp. diffère de P. mansourensis El-Naggar, 1987, P. auratum Bassock Bayiha, Nack \& Pariselle, 2016, P. combesi Bassock Bayiha, Nack \& Pariselle, 2016 et P. valentini Bassock Bayiha, Nack \& Pariselle, 2017 par : 1) un pénis filiforme beaucoup plus court chez P. essetchii n. sp. $(154,4$ vs $325,180,228,482 \mu \mathrm{m}$ respectivement chez $P$. mansourensis, $P$. auratum, $P$. combesi et $P$. valentini); 2 ) un vagin en forme de V chez $P$. essetchii n. sp. ouvert dans sa partie distale et qui présente une seule spire dans sa partie

MOTS CLÉS

Monogènes, Protoancylodiscoides,

Afrique,

Clarotes,

espèces nouvelles. proximale vs plusieurs spires chez $P$. mansourensis (4 à 5), $P$. combesi (2 à 3) et $P$. valentini (3 à 4). $P$. ivoiriensis $\mathrm{n}$. sp. diffère de ses congénères précédemment décrits, par la morphologie des crochets dorsaux et ventraux et par l'absence d'un onchium dans le hapteur. Les crochets dorsaux très grands ne présentent pas une garde courbée dans la partie terminale, les ventraux, contrairement aux autres espèces, se caractérisent par une longue garde et un long manche bien différenciés. 


KEY WORDS
Monogenea,
Protoancylodiscoides,
Africa,
Clarotes,
new species.

\begin{abstract}
Two new Protoancylodiscoides Paperna, 1969 (Monogenea, Ancyrocephalidae) gill parasites from Clarotes laticeps (Rüppell, 1829) (Siluriformes, Claroteidae) in Côte d'Ivoire.

The study of the gill parasite monogeneans of twenty specimens of Clarotes laticeps (Rüppell, 1829) (Siluriformes, Claroteidae) caught with gillnets in the Bagoué River (Côte d'Ivoire) revealed the presence of two new monogeneans species of the genus Protoancylodiscoides Paperna, 1969: Protoancylodiscoides essetchii $\mathrm{n}$. sp. and Protoancylodiscoides ivoiriensis $\mathrm{n}$. sp. These new species are different from the other Protoancylodiscoides species already described mainly in the morphology of the copulatory organ for $P$. essetchii n. sp. and the morphology of the dorsal and ventral anchors for $P$. ivoiriensis n. sp. P. essetchii n. sp. differs from P. mansourensis El-Naggar, 1987, P. auratum Bassock Bayiha, Nack \& Pariselle, 2016, P. combesi Bassock Bayiha, Nack \& Pariselle, 2016 and P. valentini Bassock Bayiha, Nack \& Pariselle, 2017: 1) the filiform penis is much shorter in P. essetchii n. sp. (154.4 vs $325,180,228,482 \mu \mathrm{m}$ respectively in $P$. mansourensis, P. Auratum, P. combesi and $P$. valentini); and 2 ) the vagina, generally V-shaped in $P$. essetchii n. sp., is open distally. Vagina has a single turn in its proximal part vs multiple turns in $P$. mansourensis (4 à 5), $P$. combesi (2 à 3 ) and $P$. valentini (3 à 4). Protoancylodiscoides ivoiriensis $\mathrm{n}$. sp. differs from all its congeners previously described in this genus, mainly by the morphology of the dorsal and ventral anchors and the non-existence of medial-dorsal onchium and medial-ventral onchium in the haptor. The very large dorsal anchors do not have a curved guard in the terminal part. The ventral anchors, unlike the other species already described in this genus, are characterized by a long guard and a well differentiated long shaft.
\end{abstract}

\section{INTRODUCTION}

Les monogènes du genre Protoancylodiscoides Paperna, 1969, parasites branchiaux des poissons Siluriformes de la famille des Claroteidae Risch, 1986 et Malapteruridae Norris, 2002 ont fait l'objet de plusieurs études (Paperna 1969; El-Naggar 1987; Euzet et al. 1989; Bilong Bilong et al. 1997; N’Douba \& Lambert 1999; N'Douba 2000; Bassock et al. 2016, 2017). En effet, Paperna (1969), au Ghana, décrit Protoancylodiscoides chrysichthes Paperna, 1969 chez Chrysichthys nigrodigitatus (Lacépède, 1803). En Egypte, El-Naggar (1987) décrit Protoancylodiscoides mansourensis El-Naggar, 1987 chez Chrysichthys auratus (Geoffroy Saint-Hilaire, 1808), cette même espèce a été retrouvée par Obiekezie et al. (1988) chez C. nigrodigitatus. Avant 1997, le genre Protoancylodiscoides était rapporté comme parasite exclusif du genre Chrysichthys Bleeker, 1858 (Siluriformes, Claroteidae). Bilong-Bilong et al. (1997), au Cameroun, révèlent chez les Malapteruridae, la présence d'une espèce du genre Protoancylodiscoides: P. malapteruri BilongBilong, Birgi \& Le Brun, 1997 chez Malapterurus electricus (Gmelin, 1789). En Côte d'Ivoire, N'Douba \& Lambert (1999), décrivent Protoancylodiscoides katii N'Douba \& Lambert, 1999 sur les branchies de Malapterurus electricus. Au Cameroun, Bassock et al. (2016) signalent deux nouvelles espèces : Protoancylodiscoides auratum Bassock, Nack \& Pariselle, 2016 et Protoancylodiscoides combesi Bassock, Nack \& Pariselle, 2016 chez C. auratus. Toujours au Cameroun, Bassock et al. (2017) décrivent Protoancylodiscoides sanagaensis Bassock, Nack \& Pariselle, 2017 chez Chrysichthys nigrodigitatus et Chrysichthys longidorsalis Risch \& Thys van den Audenaerde, 1981 ; Protoancylodiscoides valentini Bassock, Nack \& Pariselle, 2017 chez C. nigrodigitatus et C. longidorsalis; et Protoancylodiscoides spirovagina Bassock, Nack \& Pariselle, 2017 chez Chrysichthys nigrodigitatus.
En Côte d'Ivoire nos recherches sur les parasites branchiaux des poissons Siluriformes de la rivière Bagoué nous ont permis d'observer deux nouvelles espèces de monogènes du genre Protoancylodiscoides, que nous décrivons ci-dessous.

\section{MATÉRIEL ET MÉTHODES}

Les 20 spécimens de poissons Clarotes laticeps (Rüppell, 1829), d'une longueur totale de $155-356 \mathrm{~mm}$, utilisés dans cette étude ont été capturés au filet maillant dans la rivière Bagoué d'août 2018 à janvier 2019 au niveau des stations de Samorossoba $\left(9^{\circ} 52^{\prime} \mathrm{N}, 6^{\circ} 21^{\prime} \mathrm{O}\right)$; Samorosso (9⒊ $\left.34^{\prime} \mathrm{N}, 6^{\circ} 30^{\prime} \mathrm{O}\right)$ et de Guinguéreni $\left(9^{\circ} 32^{\prime} \mathrm{N}, 6^{\circ} 35^{\prime} \mathrm{O}\right)$ (Fig. 1). Ces poissons ont été immédiatement identifiés à l'aide de la clé de Paugy et al (2003). Les branchies disséquées par deux sections, l'une ventrale et l'autre dorsale, ont été étiquetées en fonction des poissons, puis conservées dans de l'azote liquide. Au laboratoire, après décongélation, les arcs branchiaux ont été séparés et placés dans une boîte de Pétri. Par un rinçage intense, les monogènes ont été détachés des branchies. Ils ont ensuite été prélevés individuellement, à l'aide d'aiguilles d'entomologie, puis transférés directement sur une lame dans une microgoutte de mélange picrate d'ammonium-glycérine (Malmberg 1957). La lame a été ensuite recouverte d'une lamelle et, après diffusion complète de la solution de montage, la préparation a été lutée avec du Glyceel et étiquetée conformément à la branchie. Un microscope (Motic BA310) muni d'une caméra intégrée a été utilisé pour les observations, l'identification et les captures d'images. Les mensurations et les dessins (Fig. 2) ont été réalisés respectivement à l'aide des logiciels Motic image 2.0 et Corel DRAW (version 20.1.0.707). Les mensurations ont été effectuées selon Gussev (1962), modifié par N'Douba (2000). Toutes les mesures (moyenne, minimum et maximum) 


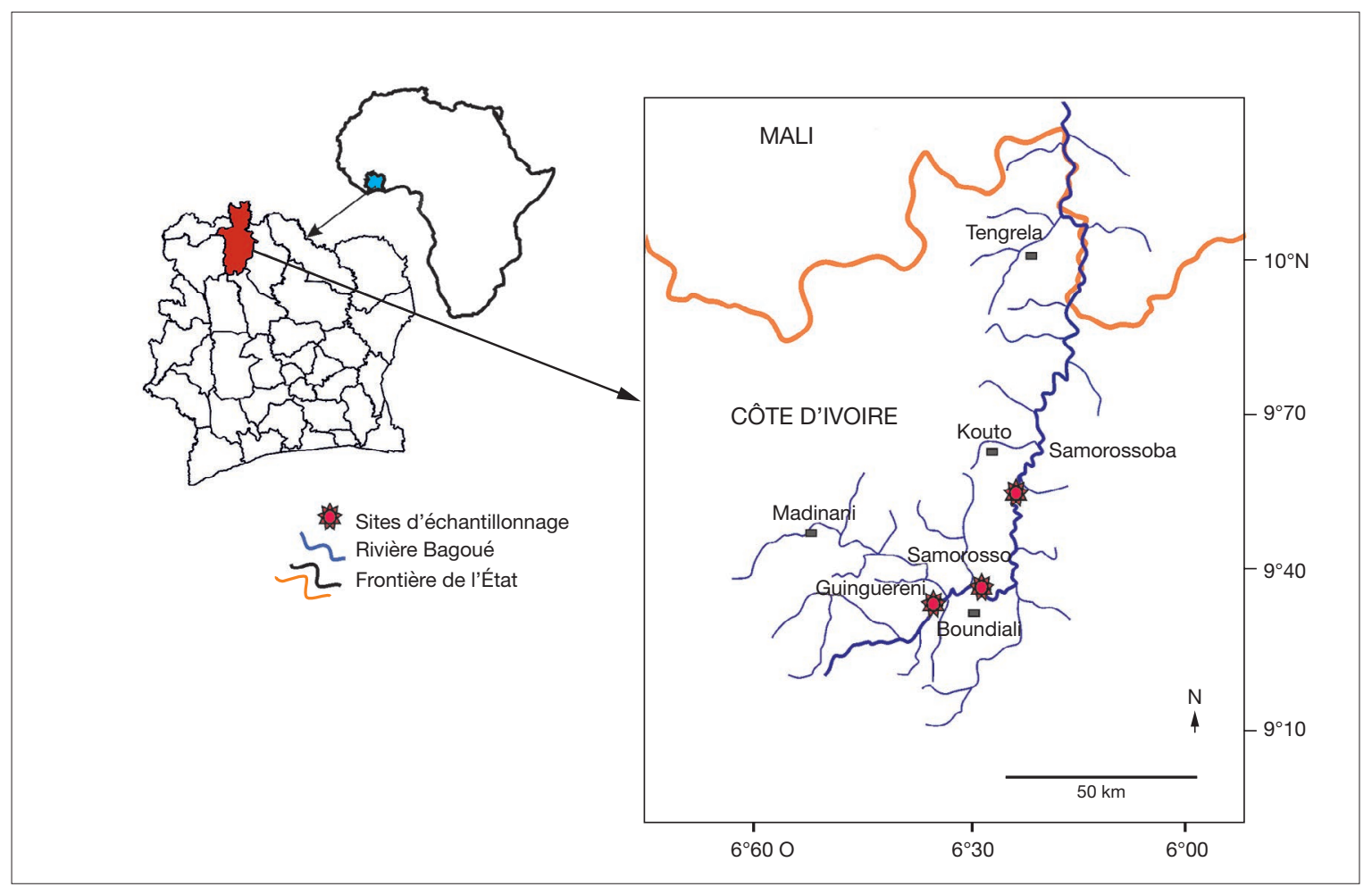

FIG. 1. - Localisation de la rivière Bagoué et des stations d'échantillonnage.

sont exprimées en micromètres $(\mu \mathrm{m})$. La dénomination des différentes pièces sclérifiées du hapteur suit Pariselle $\&$ Euzet (1995). La numérotation des crochetons a été celle adoptée à ICOPA IV (Euzet \& Prost 1981). L'intensité moyenne et la prévalence ont été définies d'après Bush et al (1997).

\section{ABRÉVIATIONS \\ MNHN Muséum national d'Histoire naturelle, Paris; \\ $\mathrm{MZH} \quad$ Finnish Museum of Natural History, Helsinki.}

\section{RÉSULTATS}

Classe MONOGENEA Carus, 1863

Ordre DACTYLOGYRIDEA Bychowsky, 1933

Famille ANCYROCEPHALIDAE Bychowsky, 1937

Genre Protoancyrodiscoides Paperna, 1969

ESPÈCE TYPE. - Protoancyrodiscoides chrysichthys Paperna, 1969.

DiAGNOSE DU GENRE. - Ancyrocephalidae : hapteur avec quatre crochets (deux dorsaux, deux ventraux); deux barres transversales (une dorsale, une ventrale composée de deux sclérites jointifs en forme de V); quatorze crochetons; un ou deux onchia présents ou absents; trois paires de glandes céphaliques; deux paires de taches oculaires sans cristallin; branches intestinales unies postérieurement; testicule postérieur intercaecal; canal déférent entourant la branche intestinale gauche; une vésicule séminal; pénis tubulaire avec pièce accessoire entourant liextrémité distale; un réservoir prostatique; ovaire médian pré-testiculaire; ouverture vaginale latérale gauche; canal vaginal contourné ou non; réceptacle séminal présent. Parasites des poissons siluriformes de la famille des Claroteidae et des Malapteruridae.

\section{Protoancylodiscoides essetchii $\mathrm{n}$. sp.}

(Fig. 3)

\section{urn:Isid:zoobank.org:act:A9378DDC-B17B-4B22-895E-998E3BB734C6}

HÔTES TYPES. — Clarotes laticeps (Rüppell, 1829).

ÉTYMOLOGIE. - “essetchii” en hommage au professeur Paul Essetchi Kouamélan, directeur de l'unité de formation et de recherche (UFR)

Biosciences de l'université Felix Houphouët-Boigny de Cocody, en Côte d'Ivoire.

SPÉCIMENS ÉTUdiés. - 25 individus montés dans la solution de Malmberg.

SPÉCIMENS TYPES. - Holotype. Côte d'Ivoire • Rivière Bagoué, Samorossoba; 952’'N, 6²1'O; 24.X.2018; Bouah Enoutchy Fabrice leg.; MNHN-HEL-1353.

Paratypes. Côte d'Ivoire 11 spécimen; mêmes données que pour l'holotype; MNHN-HEL-1354 • 2 spécimens; mêmes données que pour l'holotype; MZH, http://id.luomus.fi/KV.656 \& http:// id.luomus.fi/KV.657.

HABITAT. - Branchies.

LOCALITÉ TYPE. — Rivière Bagoué, Samorossoba (952’N, 6²1'O).

Autre localité. - Samorosso $\left(9^{\circ} 34^{\prime} \mathrm{N}, 6^{\circ} 30^{\prime} \mathrm{O}\right)$ et Guinguereni $\left(9^{\circ} 32^{\prime} \mathrm{N}, 6^{\circ} 35^{\prime} \mathrm{O}\right)$.

PRÉVAlenCe (\%). -100.

INTENSITÉ MOYENNE. - 14,5.

DiAGNOSE. - Protoancylodiscoides essetchii n. sp. se rapproche, par la morphologie des pièces sclérifiées du hapteur, de $P$. mansourensis, $P$. auratum, $P$. combesi et $P$. valentini. Toutes ces espèces se caracté- 

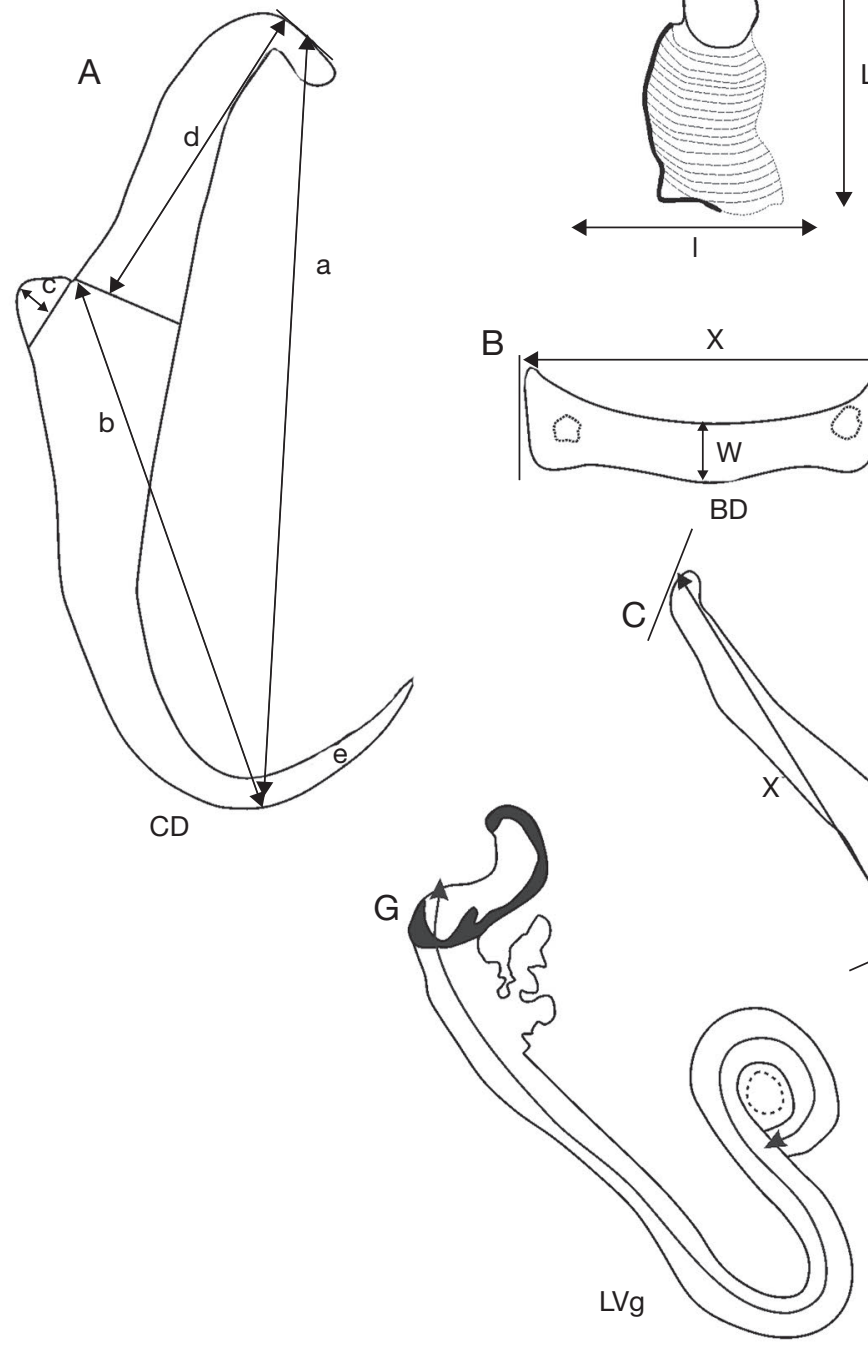

B
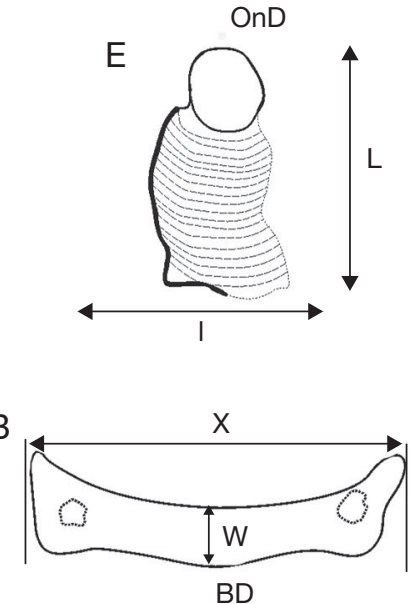

F

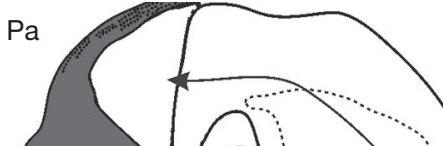

D

c)

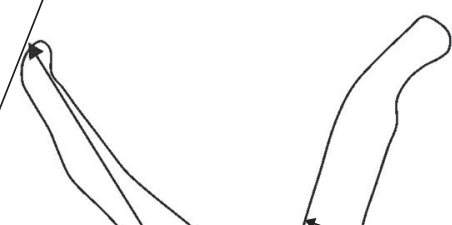

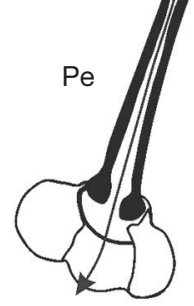

FIG. 2 - Mesures utilisées dans cette étude : A, crochet dorsal (CD), a, longueur totale, b, longueur de la lame, $\mathbf{c}$, longueur du manche, $\mathbf{d}$, longueur de la garde, e, longueur de la pointe; B, barre transversale dorsale (BD), $\mathbf{x}$, longueur, w, largeur; C, barre ventrale (BV) : x, longueur, w, largeur; $\mathbf{D}$, longueur des crochetons (U) ; E, onchium dorsal (OnD) : L, longueur, I, largeur; F, longueur du pénis $(\mathbf{P e})$, pièces accessoires $(\mathbf{P a})$; $\mathbf{G}$, longueur du vagin (LVG).

risent par des crochets dorsaux de grandes tailles, avec une longue garde repliée dans la partie terminale, un court manche; des crochets ventraux plus petits que les dorsaux et présentant des orifices à la base de la garde. Elles sont également proches par la morphologie de la barre transversale ventrale en forme de $V$ plus ou au moins ouverte et par la présence d'un onchium médio-dorsal. Cependant, $P$. essetchii n. sp. diffère fondamentalement de $P$. mansourensis, $P$. auratum, $P$. combesi et $P$. valentini par la morphologie ou la taille des organes copulateurs mâle et femelle : 1) le pénis filiforme est beaucoup plus court chez $P$. essetchii n. sp. $(154,4 \mu \mathrm{m}$ vs $325 \mu \mathrm{m}$; $180 \mu \mathrm{m} ; 228 \mu \mathrm{m} ; 482 \mu \mathrm{m}$ respectivement chez $P$. mansourensis, $P$. auratum, $P$. combesi et $P$. valentini); 2 ) le vagin généralement en forme de V chez P. essetchii n. sp. présente une seule spire dans sa partie proximale vs plusieurs spires ( 4 à $5 ; 2$ à 3 et 3 à 4 ) respectivement chez $P$. mansourensis, $P$. combesi et $P$. valentini.

\section{DESCRIPTION}

Taille des adultes : 857 (706-1023) $\mu \mathrm{m}$ sur $133(97-162) \mu \mathrm{m}$ de largeur, au niveau de l'ovaire. Crochets dorsaux très grands $[\mathrm{a}=65(56-69) \mu \mathrm{m} ; \mathrm{b}=47(44-50) \mu \mathrm{m}, \mathrm{c}=4(2-5) \mu \mathrm{m} ; \mathrm{d}=$ $25(21-28) \mu \mathrm{m} ; \mathrm{e}=21(18-23) \mu \mathrm{m}]$, garde repliée dans sa partie terminale, 6,9 fois plus longue que le manche très court, 


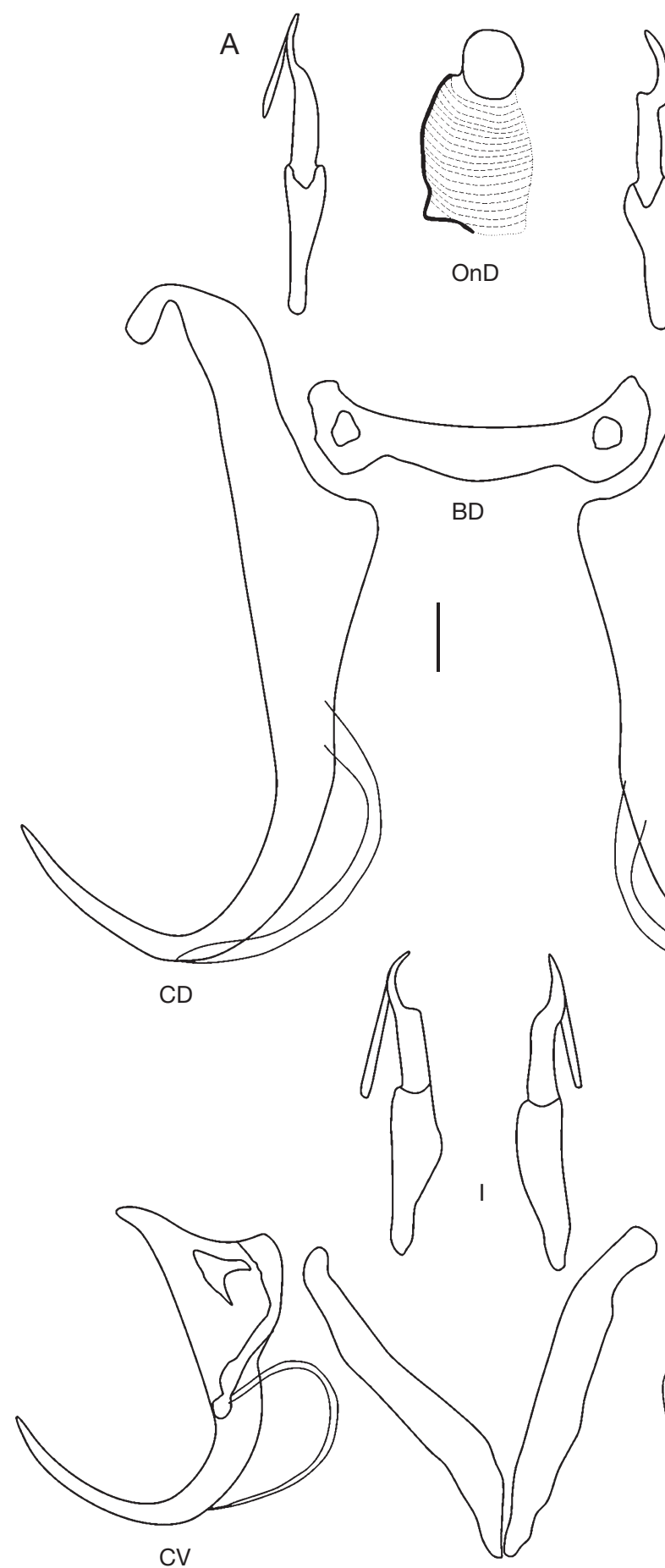

BV
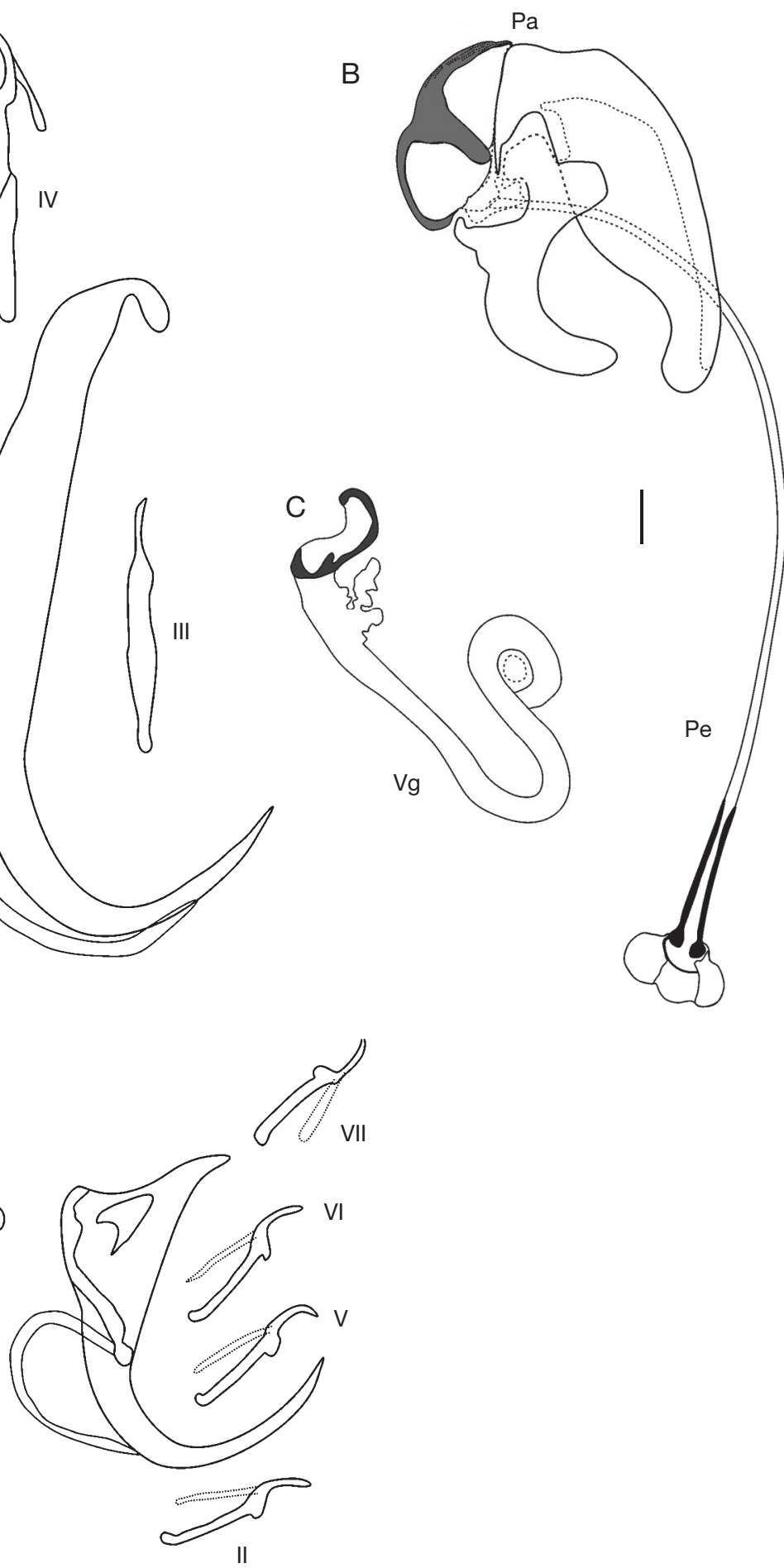

VII

FIG. 3. - A, pièces sclérifiées de Protoancylodiscoides essetchii n. sp. : barre dorsale (BD), barre ventrale (BV), crochet dorsal (CD), crochet ventral (CV), crochetons (I à VII), onchium dorsal (OnD); B, organes copulateurs de Protoancylodiscoides essetchii n. sp. : organe copulateur mâle, pièce accessoire (Pa); pénis $(\mathbf{P e})$; C, vagin $(\mathbf{V g})$. Échelles : $10 \mu \mathrm{m}$.

lame longue et arquée. Barre transversale dorsale simple, avec des extrémités légèrement élargies $\mathrm{x}=33(29-35) \mu \mathrm{m} ; \mathrm{w}=6$ (5-6) $\mu \mathrm{m}$. Crochets ventraux $[\mathrm{a}=32(31-34) \mu \mathrm{m} ; \mathrm{b}=30$ (25-32) $\mu \mathrm{m} ; \mathrm{c}=2(2-3) \mu \mathrm{m} ; \mathrm{d}=16(12-18) \mu \mathrm{m} ; \mathrm{e}=19$ (1621) $\mu \mathrm{m}$ ], deux fois plus petits que les dorsaux, garde longue, manche court, avec une carène à extrémité renflée dans la partie concave de la lame, filament de la carène coiffant la lame. Barre transversale ventrale, avec deux barres jointives en forme de $\mathrm{V} x=34(32-36) \mu \mathrm{m} ; \mathrm{w}=6(4-6) \mu \mathrm{m}$. Crochetons I, III et IV [28 (23-31) $\mu \mathrm{m} ; 24(20-27) \mu \mathrm{m} ; 31(28-32) \mu \mathrm{m}]$, plus 


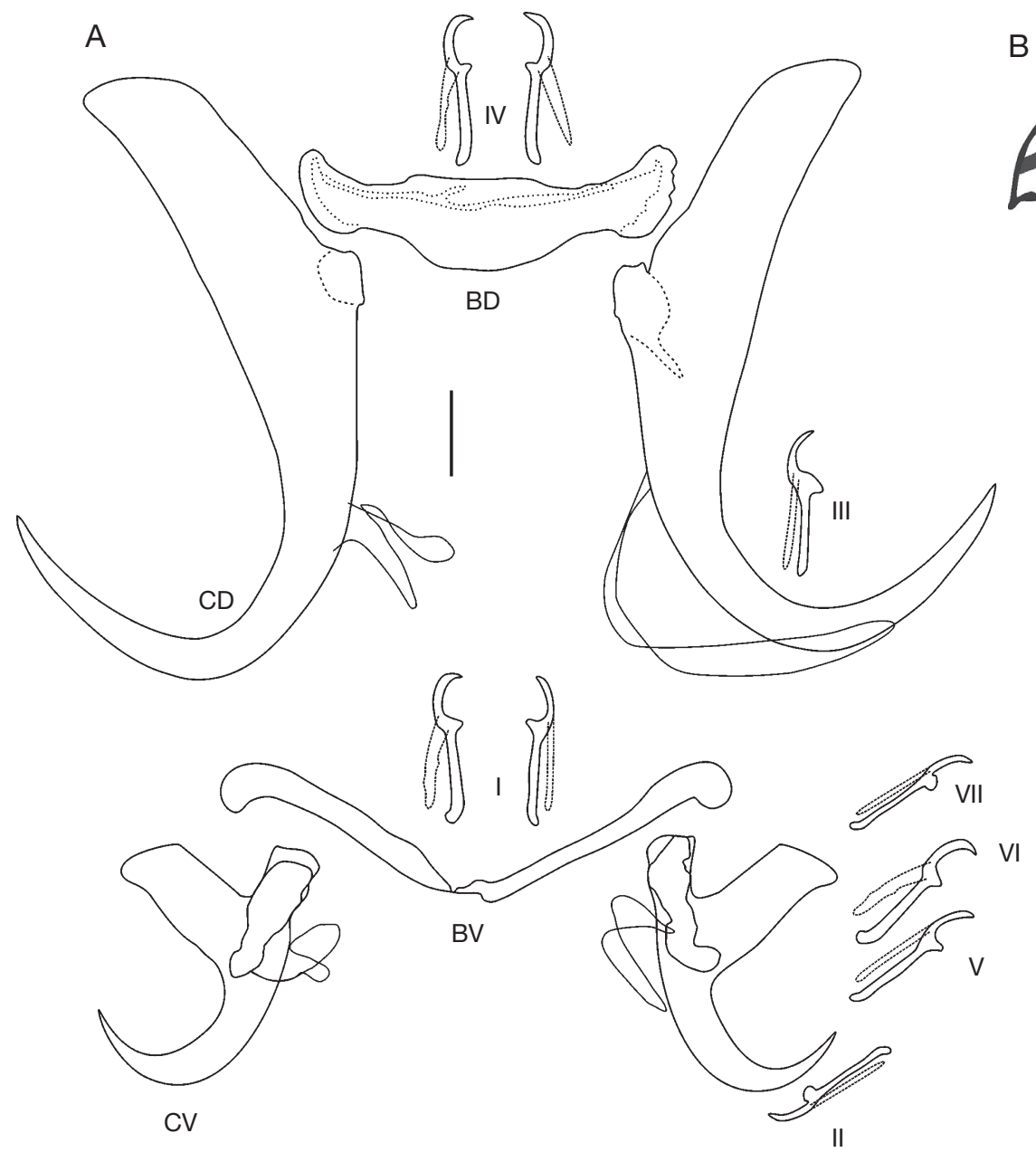

B $\mathrm{Pa}$
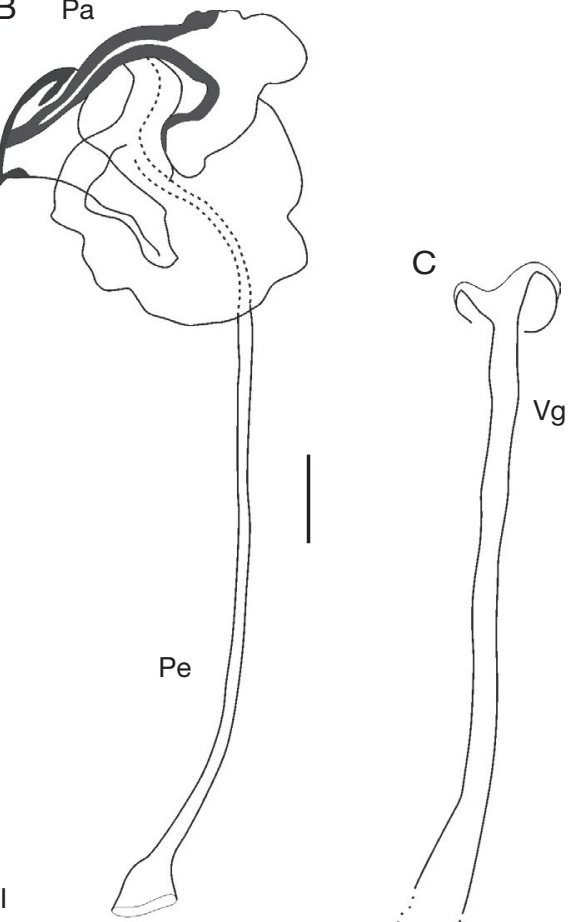

FIG. 4. - A, pièces sclérifiées de Protoancylodiscoides ivoiriensis n. sp. : barre dorsale (BD), barre ventrale (BV), crochet dorsal (CD), crochet ventral (CV), crochetons (I à VII), onchium dorsal (OnD); $\mathbf{B}$, organes copulateurs de Protoancylodiscoides ivoiriensis n. sp. : organe copulateur mâle, pièce accessoire (Pa), pénis $(\mathbf{P e})$; C, vagin $(\mathbf{V g})$. Échelles : $10 \mu \mathrm{m}$

longs que les crochetons II, V, VI, VII de longueur comprise entre 14 et $16 \mu \mathrm{m}$. Onchium médio-dorsal présent $\mathrm{L}=20$ $(16-22) \mu \mathrm{m} ; 1=15(11-18) \mu \mathrm{m}$. Pénis, tubulaire, long et filiforme $\mathrm{Pe}=154(114-190) \mu \mathrm{m}$. Pièce accessoire complexe $\mathrm{Pa}$ $=68(51-83) \mu \mathrm{m}$. Vagin en forme de $\mathrm{V}$, ouvert dans sa partie distale $\operatorname{Lvg}=96(77-109) \mu \mathrm{m}$.

\section{Protoancylodiscoides ivoiriensis n. sp.}

(Fig. 4)

\section{urn:Isid:zoobank.org:act:0A8CFD10-BF11-4FFB-92C5-2F303A944286}

HOOTES TYPES. — Clarotes laticeps (Rüppell, 1829).

ÉTYMOLOGIE. — “ivoiriensis"en référence à la Côte d'Ivoire.

SPÉCIMENS ÉTUDIÉS. - 20 individus montés dans la solution de Malmberg.
SPÉCIMENS TYPES. - Holotype. Côte d'Ivoire • Rivière Bagoué, Samorossoba; 952’N, 621'O; 24.X.2018; Bouah Enoutchy Fabrice leg.; MNHN-HEL-1355.

Paratypes. Côte d'Ivoire 1 specimen; mêmes données que pour l'holotype; MNHN-HEL-1356 • 1 specimen; mêmes données que pour l'holotype; MZH, http://id.luomus.fi/KV.658.

HABITAT. - Branchies.

LOCALITÉ TYPE. - Rivière Bagoué, Samorossoba (952’ N, 6²1’O).

Autre localité. - Samorosso (9³4’ N, 6³0’O).

PRÉVALENCE (\%). -25 .

INTENSITÉ MOYENNE. -7 .

DiAGNOSE. - Cette espèce differe de toutes ses congénères précédemment décrites dans ce genre, principalement par la morphologie des crochets dorsaux et ventraux et par l'absence d'onchium dans le hapteur. Les crochets dorsaux larges, ne présentent pas une garde courbée dans la partie terminale. Les crochets ventraux, contraire- 
ment aux autres espèces déjà décrites dans ce genre, se caractérisent par une longue garde et un long manche bien différenciés. Les crochetons I, III, et IV, à la différence de ce que l'on observe chez les autres espèces du genre, ont conservés leur taille larvaire tout comme c'est le cas pour les crochetons II, V, VI, VII.

\section{DESCRIPTION}

Taille des adultes : 1093 (623-1429) $\mu$ m sur 215 (139302) $\mu \mathrm{m}$ de largeur, au niveau de l'ovaire. Crochets dorsaux larges, garde longue, manche très court, lame longue, arquée à longue pointe, $\mathrm{a}=61(55-65) \mu \mathrm{m} ; \mathrm{b}=50(43-51) \mu \mathrm{m}$; $\mathrm{c}=3(3-4) \mu \mathrm{m} ; \mathrm{d}=28(24-31) \mu \mathrm{m} ; \mathrm{e}=28(23-31) \mu \mathrm{m}$. Barre transversale dorsale légèrement incurvée, plus ou moins renflée dans sa partie médiane avec des extrémités très épaisses $\mathrm{x}=29(27-34) \mu \mathrm{m} ; \mathrm{w}=3(2-4) \mu \mathrm{m}$. Crochets ventraux plus petits que les dorsaux, garde longue, manche long, parcouru par une carène à filament coiffant une lame arquée, à pointe courte, $\mathrm{a}=27(24-28) \mu \mathrm{m} ; \mathrm{b}=23$ (21-24) $\mu \mathrm{m} ; \mathrm{c}=7(6-8) \mu \mathrm{m} ; \mathrm{d}=16(15-18) \mu \mathrm{m} ; \mathrm{e}=12$ (9-14) $\mu \mathrm{m}$. Barre transversale ventrale, en forme de $\mathrm{V}$ avec de deux barres jointives aux extrémités légèrement renflées, $\mathrm{x}=29(27-34) \mu \mathrm{m} ; \mathrm{w}=3(2-4) \mu \mathrm{m}$. Crochetons I, II, III, IV, V, VI, VII tous a l'état larvaire, longueur comprise entre 15 et $17 \mu \mathrm{m}$. Pénis long et filiforme Pe $=103$ (74116) $\mu \mathrm{m}$. Pièce accessoire complexe $\mathrm{Pa}=44$ (35-50) $\mu \mathrm{m}$. Vagin $\mathrm{Vg}=[\mathrm{n}=5,77(85,5-68) \mu \mathrm{m}]$ légèrement sclérifié, long et tubulaire, avec une ouverture en forme d'entonnoir dans sa partie distale.

\section{DISCUSSION}

La famille des Claroteidae comprend actuellement en Afrique de l'Ouest cinq genres : Clarotes Kner, 1855, Chrysichthys Bleeker, 1858, Auchenoglanis Günther, 1865, Notoglanidium Günther, 1902 et Parauchenoglanis Boulenger, 1911 (Paugy et al. 2003). Selon Paugy et al. (2003), l'espèce que nous avons étudiée, Clarotes laticeps (Rüppell, 1829), est connue dans les bassins nilo-soudaniens (Tchad, Bénoué, Niger, Sénégal, Volta).

La description de deux nouvelles espèces de Protoancylodiscoides chez cet hôte porte actuellement à 11 le nombre d'espèces qui composent ce genre de monogène (Paperna 1969; El-Naggar 1987; Euzet et al. 1989; Bilong Bilong et al. 1997; N’Douba \& Lambert 1999; Bassock et al. 2016, 2017; Scholz et al. 2018).

Parmi celles-ci :

- sept se trouvent chez les poissons du genre Chrysichthys (Claroteidae), et sont toutes caractérisées par la présence d'un onchium médio-dorsal dans le hapteur (Paperna 1969; ElNaggar 1987; Euzet et al. 1989; N’Douba 2000; Bassock et al. 2016, 2017);

- deux sont présentes chez des hôtes du genre Malapterurus Lacépède, 1803 (Malapteruridae), probablement à la suite d'un transfert latéral depuis un Bagridae (Bilong Bilong et al. 1997); elles sont caractérisées par la présence de deux onchia dans le hapteur, l'un en position médio-dorsal et l'autre en position médio-ventral (Bilong Bilong et al. 1997; N’Douba \& Lambert 1999).
Nous observons donc une certaine spécificité des parasites vis à vis de leurs hôtes (homogénéité morphologique des espèces présentes chez les genres Chrysichthys ou Malapterurus) qui doit illustrer une évolution de type vicariante suite au transfert latéral des parasites entre deux familles d'hôtes différentes.

En revanche, chez les deux nouvelles espèces que nous venons de décrire sur un Clarotes (Claroteidae), P. essetchii n. sp. a, tout comme les monogènes des Chrysichthys, un onchium médio-dorsal dans le hapteur, alors que $P$. ivoiriensis n. sp. n'en a aucun. Chez le genre Clarotes, le parasitisme est donc marqué par une coexistence de deux morphes (sans ou avec un seul onchium) dans le hapteur.

Seule une étude génétique pourra nous permettre de déterminer quelle est la forme ancestrale (absence, présence d'un ou deux onchia dans le hapteur) et donc de donner un scénario co-évolutif pour les Protoancylodiscoides et leurs hôtes.

Ce même type de co-évolution (vicariance, transfert latéral) semble avoir aussi prévalu chez un autre genre de la même famille, Auchenoglanis (Claroteidae), chez qui ont été décrits des monogènes du genre Bagrobdella Paperna, 1969 typiques des espèces de ce genre d'hôte et proche de Protoancylodiscoides (Euzet \& Le Brun 1990); et du genre Quadriacanthus Paperna 1961 suite à un transfert latéral depuis une espèces hôte de la famille des Clariidae chez qui sont généralement décrites les espèces de ce genre de monogènes (Akoumba et al. 2017).

Le parasitisme des Claroteidae mériterait donc d'être étudié davantage car, contrairement aux autres Siluriformes (Schilbeidae De Vos, 1984, Mochokidae Paugy, 1987, Clariidae Teugels, 1982 et Bagridae Risch, 1987, Malapteruridae Norris, 2002) chez qui le parasitisme par un genre donné est caractéristique et spécifique à toute la famille, celui observé chez Clarotes, Chrysichthys et Auchenoglanis, appartenant tous à la famille Claroteidae, présente une spécificité propre à chaque genre dans cette famille : Clarotes \& Chrysichthys sont parasités uniquement par le genre Protoancylodiscoides; Auchenoglanis est non seulement parasité par le genre Bagrobdella, mais aussi par le genre Quadriacanthus. Pourquoi un tel parasitisme chez les Claroteidae? La famille des Claroteidae est-elle monophylétique?

\section{Remerciements}

Les auteurs remercient le Dr Gogbe Zeré Marius, de l'Université Peleforo Gon Coulibaly de Korhogo (Côte d'Ivoire), pour nous avoir aidé et guidé tout au long de notre échantillonnage. Nous remercions M. Risto Väinölä pour avoir facilité le dépôt des différent paratypes au Musée finlandais d'Histoire naturelle (Helsinki). Les auteurs remercient également le Pr Maarten Vanhove et le Pr Jean-Lou Justine pour leurs relectures et leurs corrections.

\section{RÉFÉRENCES}

Akoumba J. F., Pariselle A., Tombi J. \& Bilong C. F. B. 2017. Description of two new ancyrocephalid (Quadriacanthus and Bagrobdella) Monogenea from the gills of auchenoglanid catfishes (Pisces, Siluriformes, Claroteidae) in Cameroon. Vie Milieu 67 (2): 59-64. 
Bassock B. E.D, Nack J., Bitja Nyom A. R., Pariselle A \& Bilong BILONG C. F. 2017. - Description of Three New Species of Protoancylodiscoides (Monogenea, Ancyrocephalidae) Gill Parasites from Chrysichthys nigrodigitatus and Chrysichthys longidorsalis (Siluriformes, Claroteidae) in the Sanaga River (Cameroon). Vie et Milieu 67 (2): 65-73.

Bassock B. E. D., Nack J., Pariselle A. \& Bilong Bilong C. F. 2016. - Two new species of gill parasites assigned to Protoancylodiscoides (Monogenea, Ancyrocephalidae) from Chrysichthys spp. (Siluriformes, Claroteidae) in River Sanaga (Cameroon). Zootaxa 4170 (1): 178-186. https://doi.org/10.11646/zootaxa.4170.1.11

Bilong Bilong C. F., Birgi E. \& Le Brun N. 1997. — Protoancylodiscoides malapteruri n. sp. (Monogenea, Dactylogyridea, Ancyrocephalidae), parasite branchial de Malapterurus electricus Gmelin (Siluriformes, Malapteruridae) au Cameroun. Systematic Parasitology 38: 203-210. https://doi.org/10.1023/A:1005892503336

Bush A. O., Lafferty D. K., Lotz M. J. \& Shostak W. A. 1997. — Parasitology meets ecology on its own terms: Margolis et al. Revisited. Journal of Parasitology 83: 575-583. https://doi. org/10.2307/3284227

El-Naggar M. M. 1987. - Protoancylodiscoides mansourensis n. sp. a monogenean gill parasite of the Egyptian freshwater fish Chrysichthys auratus Geoffroy, 1809. Arab Gulf Journal of Scientific Research, Agricultural and Biological Science, B 5: 441-454.

EUZET L., AgNÈsE J. F. \& LAMBERT A. 1989. - Valeur des parasites comme critère d'identification de l'espèce hôte. Démonstration convergente par l'étude parasitologique des Monogènes branchiaux et l'analyse génétique des Poissons hôtes. Comptes Rendus de l'Académie des Sciences de Paris, 308: 385-388.

EuZeT L. \& Prost M. 1981. - Report of the meeting on Monogenea: problems of systematics, biology and ecology, in Slusarski W. (ed.), Review of Advanced in parasitology. Warszawa Varsovie, P.W.N: polish scientific publishers: 1003-1004.

EUZET L. \& LE BRUN N. 1990. — Monogènes du genre Bagrobdella Paperna, 1969 parasites branchiaux d'Auchenoglanis occidentalis (Cuvier et Valenciennes, 1840) (Teleostei, Siluriformes, Bagridae). Journal of African Zoology 104: 37-48.
Gussev A. V. 1962. — Order Dactylogyridea, in ByChovskayaPavlovskaya I. E., Gussev A. V., Dubinina M. N., Izymova N. A., Smirnova T. S., Sokolovskaya I. L., Shtein G. A., SHUL'MAN S. S. \& EPSTHEIN V. M. (eds), Key to the parasites of freshwater fish of the USSR. Israel Program for Scientific Translations, Jerusalem. (Russian original: Opredelitel' parazitov presnovohnyh ryb SSSR. Izadtel'stovo Akademii Nauk SSSR, Moscow-Leningrad): 204-342.

MALMBERG G. 1957. - On the occurrence of Gyrodactylus on Swedish fishes. Skrifter Utgivna av Sodra Sveriges Fiskeriforening Arsskrift 1956: 19-76 (In Swedish with English abstract and species descriptions).

N'DOUBA V. \& LAMBERT A. 1999. - Un nouveau monogène du genre Protoancylodiscoides Paperna, 1969 (Ancyrocephalidae) parasite branchial de Malapterurus electricus (Gmelin, 1789) (Siluriforme), en Côte d'Ivoire. Zoosystema 21 (3): 418-421.

N’Douba V. 2000. - Biodiversité des monogènes parasites des poissons d'eau douce de Côte d'Ivoire : cas des poissons des rivières Bia et Agnébi. Doctorat Thèses, Université de Cocody, Abidjan, Côte d'Ivoire, 255 p.

ObiekeZie A. I., Muller H. \& ANDERS K. 1988. — Diseases of the African estuarine catfish Chrysichthys nigrodigitatus (Lacepède) from the Cross-River estuary, Nigeria. Journal of Fish Biology 32: 207-221. https://doi.org/10.1111/j.1095-8649.1988.tb05354.x

PAPERNA I. 1969. - Monogenetic trematodes of fish of the Volta basin and south Ghana. Bulletin de l'Institut française d'Afrique Noire 31: 840-880.

PARISElle A. \& EuZeT L. 1995. - Gill parasites of the genus Cichlidogyrus Paperna, 1960 (Monogenea, Ancyrocephalidae) from Tilapia guineensis (Bleeker, 1862), with descriptions of six new species. Systematic Parasitology 30: 187-198.

Paugy D., Lévêque C. \& Teugels G. G. 2003. - Faune des poissons d'eaux douces et saumâtres de l'Afrique de l'Ouest, Volume 2. Paris : IRD, MRAC, MNHN.

Scholz T., Vanhove M. P. M., SMit N., JaYASUndera Z. \& GELNAR M. 2018. - A guide to the parasites of African freshwater fishes. Abc Taxa 18: 1-425.

Soumis le 23 septembre 2020 accepté le 3 décembre 2020 . publié le 30 mars 2021. 